\title{
Water electrolysers with closed and open electrochemical systems
}

Marie Francine Lagadec ${ }^{1^{*}}$, Alexis Grimaud ${ }^{1,2,3^{*}}$

${ }^{1}$ Chimie du Solide et de l’Energie, Collège de France, UMR 8260, 75231 Paris Cedex 05, France.

${ }^{2}$ Réseau sur le Stockage Electrochimique de l'Energie (RS2E), CNRS FR3459, 33 rue Saint Leu, 80039 Amiens Cedex, France.

${ }^{3}$ Sorbonne Université, Paris, France.

*email: $\quad \underline{\text { marie-francine.lagadec@college-de-france.fr }}$

alexis.grimaud@college-de-france.fr

ORCID: $\quad$ MFL $\quad 0000-0002-6214-5171$

AG $\quad 0000-0002-9966-205 X$ 


\section{Abstract}

Green hydrogen production using renewables-powered, low-temperature water electrolysers is crucial for rapidly decarbonising the industrial sector and with it many chemical transformation processes. However, despite decades of research, advances at laboratory-scale in terms of catalyst design and insights into underlying processes have not significantly resulted in urgently needed improvements in water electrolyser performance or higher deployment rates. In light of recent developments in water electrolyser devices with modified architectures and designs integrating concepts from Li-ion or redox flow batteries, we discuss practical challenges hampering water electrolyser scaling-up and large-scale deployment. We highlight the role of device architectures and designs, and how engineering concepts deserve to be integrated into fundamental research to accelerate synergies between materials science and engineering and to achieve industry-scale deployment. New devices require benchmarking and assessment in terms of their performance metrics, but also of their scalability and deployment potential. 
The rapid increase of our global energy consumption, as exemplified in 2018 with a growth of $2.3 \%$ to exceed $166 \mathrm{PWh}$, its fastest pace in the last decade, drove fossil-fuel related $\mathrm{CO}_{2}$ emissions to a record level of above $33 \mathrm{Gt}^{1}$ The growing demand for electricity is the driving force behind the rise in global energy consumption as it accounts for almost $20 \%$ of the final energy consumption, and is expected to become the dominant energy source by $2050^{2}$. To date, about $2 \%$ and $43 \%$ of our electricity consumption stems from the transportation and industrial sectors ${ }^{2}$, respectively. Fortunately, nearly half of the increased production of electricity for 2018 was from renewables ${ }^{3}$, and inexpensive and sustainably produced electricity will be available at a cost $<10$ cents per $\mathrm{kWh}^{4}$.

Increasing the share of renewable energies into a sustainable energy mix requires developing and deploying energy conversion and storage solutions at large scale. There, electrochemical systems excel in terms of versatility and scalability. Thus, numerous systems have been proposed to cover a wide range of applications, from consumer electronics to electric vehicles, grid energy storage and industrial chemical transformation processes. In the transportation sector, secondary Li-ion batteries (LIBs) can now power light-duty electric vehicles with a driving range of several hundreds of $\mathrm{km}$. Indeed, driven by materials scientists as recognized by the Nobel prize in chemistry in 2019, LIBs have flourished. Their impact far exceeds their initial purpose (portable electronics) and now covers car electrification and potentially for grid energy storage. This is explained both by a $>350 \%$ increase of LIB performance since their commercialisation in 1991 (from initially 90 Wh/kg with graphite vs. lithium cobalt oxide to $>300 \mathrm{Wh} / \mathrm{kg}$ with graphite vs. lithium nickel manganese cobalt oxide today ${ }^{5}$ ) and a drastic reduction of pack cost of $>80 \%$ 
within a decade down to $<200 \$ / \mathrm{kWh}$ in $2020^{6,7}$, as initiated by the creation of giga factories.

While hydrogen can potentially play a role in the transportation sector (e.g., powering heavy-duty vehicles ${ }^{8}$ ), the sustainable production of hydrogen has a far greater shortterm impact on decarbonising the industrial sector. ${ }^{9}$ Indeed, pure and mixed hydrogen plays a key role as an intermediate for energy-demanding chemical transformation processes such as ammonia synthesis, methanol production, and metal blanketing. ${ }^{8,9}$ At present, more than $110 \mathrm{Mt}$ of hydrogen are produced per year; $95 \%$ by $\mathrm{CO}_{2}$-emitting reforming of fossil-fuels and only $4 \%$ through electrolysis, despite it being many decades older than LIB technology. The overruling explanation for the low market penetration of water electrolysis is its hydrogen production price. Indeed, hydrogen produced by reforming of fossil-fuels comes at a cost of $1.3-1.5 \$ / \mathrm{kg}$ of $\mathrm{H}_{2}$, while green (renewablespowered) water electrolysis, now running at $>4 \$ / \mathrm{kg}$ of $\mathrm{H}_{2}$, must reach $\sim 2 \$ / \mathrm{kg}$ of $\mathrm{H}_{2}$ to become cost competitive. ${ }^{10}$

The industrial development and deployment of water electrolysers (WEs) is therefore in stark contrast to the recent success story of LIBs. This difference originates, in part, from the inherent difficulties associated with scaling up and improving the performances of open electrochemical systems compared to those of closed electrochemical systems. Europe leads with an annual electrolyser manufacturing capacity in the GW range, ${ }^{11}$ but a deployment level of 0.1-1 TW per year is needed ${ }^{12}$. Recently, we have thus seen materials and concepts originating from battery technology being applied to new WE devices, leading to some of those being labelled battolysers. ${ }^{13,14}$ While such potentially synergistic merging of concepts is exciting at a fundamental level, its practicality requires a critical evaluation which must be made in light of past and current developments of more ma- 
ture WEs technologies. This perspective thus briefly recalls the fundamental differences between architecture and design of batteries and WEs, and more specifically why their transpositions from lab-scale discoveries to industrial-scale setup are very different. Next, we expose practical limitations having hampered the development of low temperature WEs. With this in mind, we will finally introduce different design strategies recently proposed and discuss potential promises offered by these new WEs architectures and designs, as well as their complementarity with traditional electrolyser technologies.

\section{Closed versus open systems}

Electrochemical energy conversion and storage devices can be classified into closed systems (such as Li-ion, Na-ion batteries and supercapacitors, see Fig. 1a), and open systems (for instance redox flow batteries and low-temperature WEs, Fig. 1b). In the latter one, reactants and reaction products are circulated in and out of the electrochemical cells while closed systems require no flow management, which improves both their volumetric capacity and their manufacturability. More importantly, the development of closed systems thrives from the relatively direct transferability of results obtained in laboratory conditions to practical devices. Indeed, no drastic differences exist between electrochemical cells used at the laboratory-scale (say, coin cells) and industrial small-scale cell formats (cylindrical 18650 or pouch cells) (Fig. 1a), while for packs used in large scale applications such as electric vehicles, a battery management system is required to control temperature, state-of-charge and the cooling system. In both cases, electrochemically active materials - typically mixed with carbon black, binder and solvents - are directly coated onto current collectors before calendaring and rolling or stacking them with the porous separator. In contrast, for open systems, gas and/or liquid management re- 
quires specific measures at both cell and stack level (selective membranes allowing ionic transport and avoiding cross-diffusion of reactants and/or products, and porous current collectors allowing liquid or gaseous reactants to reach the heart of the electrochemical cell), and at the system level, where expensive balance-of-plant (BOP) equipment such as power electronics, external circulation, and gas processing systems is required (Fig. 1b).

The development of better components for WEs, as for any open electrochemical system, is thus a rather frustrating task for materials scientists, since optimised physical properties of the single components do not directly translate into better system performance. This is especially true for both oxygen evolution reaction (OER) and hydrogen evolution reaction (HER) electrocatalysts which are rarely tested in practical conditions. ${ }^{15-18}$ Indeed, for lab-scale evaluation of the performance of all WE technologies, the intrinsic properties and performance characteristics of the membranes (e.g., ionic conductivity) and those of the catalysts/electrodes (e.g., intrinsic and geometric activity $^{19}$ ) are typically reported as performance indicators. Thus, many recent studies have reported the development of catalysts with ever improving performance. For instance, overpotentials as low as $250 \mathrm{mV}$ at $10 \mathrm{~mA} / \mathrm{cm}^{2}$ catalyst are now reached using laboratory tests in both alkaline conditions using crystalline or amorphous transition metal oxide catalysts, and acidic conditions using Ir-based OER catalysts, while overpotentials as low as $100 \mathrm{mV}$ at $10 \mathrm{~mA} / \mathrm{cm}^{2}$ catalyst are found for transition metal sulphides or phosphides HER catalysts in acidic conditions ${ }^{20}$. These intrinsic properties are very important since they set up the performance limits at the system level as shown in Box 1. Hence, any loss of intrinsic activity (mass and surface normalised) would have to be counterbalanced by an increase in loading. This will eventually impact mass transfer and 
performance of industry-scale WEs that are in turn determined by the extrinsic properties of the membrane electrode assembly (MEA, see Fig. 2a) and its interplay with the sandwiching porous transport layers (PTLs) and bipolar plates (BPs). This directly impacts the current density that can be achieved by the overall system. An overview on intrinsic and extrinsic properties affecting stack performance and cost is given in Table $\mathbf{S} 2$ in the supplementary information (SI). Furthermore, in the MEA configuration, current densities of several hundreds of $\mathrm{mA} / \mathrm{cm}^{2}$ are applied with catalyst loadings as high as $1-2 \mathrm{mg} / \mathrm{cm}^{2}$. Proton exchange membrane water electrolysers (PEMWEs, Fig. S1c in the SI) operate at current densities $>1.6 \mathrm{~A} / \mathrm{cm}^{2}$ while alkaline water electrolysers (AWEs, Fig. S1a in the SI) operate at around $0.5 \mathrm{~mA} / \mathrm{cm}^{2}$, see Table S3 in the SI. These numbers, in view of the need to drastically decrease the loading of Ir-based OER catalysts, are in excess of what is common in laboratory conditions (current densities in the order of $\sim 10 \mathrm{~mA} / \mathrm{cm}^{2}$ and typical catalyst loadings of $0.1-0.3 \mathrm{mg} / \mathrm{cm}^{2}$, see Discussion S4 in the $\mathrm{SI}$ ) in the so-called three electrode configuration (TEC) shown in Fig. $\mathbf{2}$ b. ${ }^{21}$ Indeed, the best practice in assessing the mass and/or specific activity of catalysts ${ }^{22}$ is to use TECs with a thin catalyst layer deposited on the conductive rotating electrode substrate. Thus, bottlenecks arising from mass transport limitation (i.e., gas diffusion) and/or electrical conductivity are, at least partially, avoided by applying a rotation as well as by limiting the current density and using conductive carbon ${ }^{23}$. In addition, TECs require the use of Nafion ${ }^{\circledR}$ as a binder to ensure the mechanical stability of the electrodes. For MEAs, an ionomer is used to ensure both ionic conductivity and liquid/gas management while ensuring the mechanical stability of the catalyst; this requires a loading three orders of magnitude greater than the Nafion ${ }^{\circledR}$ loading in TECs. Thus, optimizing MEAs is a much more complex task than optimizing TECs, and must be carried out for each catalyst and/or conductive substrate. 
This difference in testing protocols results in large discrepancies in measured performances, with lab-scale results obtained via TECs often being more promising than those measured in MEA configurations. Hence, a factor of 2 to 3 in discrepancy between MEA and TEC performance was recently reported for an iridium-based OER catalyst for PEMWEs ${ }^{15}$, similar to what was previously obtained for fuel cell catalysts ${ }^{24}$. This discrepancy largely originates from the different interfaces formed in MEAs and TECs. Solid/gas interfaces are found in MEAs (Fig. 2a), while for TECs, the catalyst is dipped into a liquid electrolyte, thus forming a solid/liquid interface (Fig. 2b). ${ }^{25}$ Furthermore, prolonged tests during, e.g., $>100 \mathrm{k} h$ with current densities in the order of $A / \mathrm{cm}^{2}$ (the currently achieved performance for MEA-based systems) cannot be carried out in TECs due to chemical and mechanical stability issues related to the glassware and the catalyst, respectively. The discrepancy in test duration and stability for TECs and MEAs calls for the development of accelerated testing protocols for TECs.

\section{Performance and price evaluation}

Few research laboratories are currently equipped for assembling and testing catalysts in the MEA configuration, and only the dissemination of benchmarks can alleviate our current inability to compare catalyst activities. To speed up the pace of findings which help improve WE performance, the ability to properly evaluate performance improvements for each component is essential (Fig. 3a). Indeed, major discrepancies exist between reported lab-scale performance metrics and the parameters required to assess a demonstrator's suitability for industry-sized up-scaling, and very few methodologies exist for bridging these two worlds (see ref. ${ }^{25}$ for a detailed analysis). This difficulty in assessment becomes even more blatant when comparing catalyst stability using TECs with catalyst 
durability in MEAs, which is impacted by assembly techniques and by the specific properties of membranes and electrodes. This calls for the use of standardised materials and conditions reflecting realistic industrial operation, as recognized in the field. ${ }^{26,27}$ These include, for example, the use of high catalyst loadings on PTLs, high current densities, highly concentrated feed solutions and elevated temparatures. ${ }^{28}$ Further, accelerated stress tests are key to assessing the impact of varying operation conditions (fluctuating current density and temperature) on lifetime and performance ${ }^{29}$ and of catalyst loading on performance and durability. ${ }^{30,31}$ In our present understanding, the exact impact of physical properties of electrocatalysts on the performance and durability of practical devices still remains a conundrum to which efforts should be dedicated. In contrast, the much younger fields of $\mathrm{CO}_{2}$ or $\mathrm{N}_{2}$ electro-reduction ${ }^{32-36}$ have widely benefited from such standardised testing protocols. Indeed, researchers have recognised early on that reliable results on the selectivity of given catalysts can only be obtained by first defining adequate testing protocols and electrochemical setups, and also that efforts at improving electrolyser design, which can greatly affect the local pH for instance, directly impact catalyst selectivity and overall device performance. New electrochemical testing environments mimicking the solid/gas interface formed in MEAs and allowing the understanding of subtle local effects must therefore be developed to test HER and OER catalysts in laboratory conditions.

A breakdown of PEMWE system cost (Fig. 3a) shows that for present-day PEMWE technology at current production rates, MEA cost only accounts for $14 \%$ of system cost and $\sim 26 \%$ of stack cost. ${ }^{37}$ System cost is dictated by BOP cost $(\sim 50 \%$ and more for larger systems). Table S5 in the SI gives an overview on key information and assumptions used for the cost calculation in ref. ${ }^{37}$ and Tables S6-S7 contain the data plotted in Fig. $\mathbf{3}$. The lab- 
scale development of non-noble metal-based catalysts for anion exchange membrane water electrolysers (AEMWEs, see Box 1 and Fig. S1c in the SI) is often justified by a potential for cost reduction. However, at current low production rates, MEA cost is not limiting stack cost, even with expensive PEMWE catalysts; rather, it is determined by the cost of the PTL and BP as explained in Box 1. Thus, both for benchmarking catalyst performance and for decreasing WE cost, research efforts must be directed at developing cost-effective corrosion-resistant porous conductive networks. Towards that goal, conductive porous transition metal nitrides have been tested ${ }^{38}$, though within a limited potential range. While much more complex than the quick performance evaluation of OER or HER catalysts, the development and manufacturing of suitably structured materials can have a tremendous impact on the development and deployment of low-temperature WEs. This is particularly the case when decreasing the loading of Ir-based OER catalysts by using high-mass activity catalysts which cannot be used without conductive substrates.

One major way to decrease both system and stack cost of PEMWEs is by increasing the size/power of the system (Fig. 3b). As the relative price associated with expensive PTL and BP materials can be decreased with larger components and optimised manufacturing techniques ${ }^{39-41}$ which can halve the price of a PEMWE stack, and the same can be assumed to hold for AEMWE stacks. In doing so, the relative cost associated with the MEA will automatically increase, but the major impact of materials used for the MEA (i.e., the catalysts and the membrane) is on device scalability. More specifically, while the loading of Pt used as HER catalyst can be drastically decreased to $<0.1 \mathrm{mg} \mathrm{Pt}_{\mathrm{Pt}} / \mathrm{cm}^{2}$ without affecting overall performance ${ }^{42}$, the use of Ir as OER catalyst represents a technological bottle- 
neck. Indeed, its loading can hardly be decreased without a significant loss of performance, and the current worldwide production of less than 10 tons per year, against 100s of tons for Pt, drastically limits the deployment of PEMWE technology. ${ }^{20}$ Nevertheless, relatively limited research efforts are dedicated to the development of Ir-based OER catalysts for PEMWEs with better performances compared to OER catalysts for AEMWEs. This discrepancy is largely explained by the relative ease of quickly synthetizing and testing $3 d$ transition metal oxides such as perovskites or spinels. Most oxides, including complex Irbased ones, are unstable in acidic conditions, with the exception of $\mathrm{IrO}_{2}$ currently used in commercial PEMWEs and showing stability over 100k hours, making their intrinsic properties difficult to study and the mastering of their chemistry complex. Concerted research efforts must be aimed at this and at the development of better PTL/MEA combinations so as to directly correlate the performance and scalability of WE devices. Finally, any decrease in performance, i.e., in current density, reached in operation conditions and due catalysts with lower intrinsic performances or lower extrinsic performances of the MEA/PTL assembly, will have to be counterbalanced by an equal drop in overall cost. As system cost of open electrochemical systems such as WEs is largely dominated by BOP cost (see Fig. 3a-c), this cost reduction will be difficult to achieve, making it critical to maintain a large operational current density.

As exemplified by the massive impact of giga factories on the deployment capability and the pack cost for LIBs, ${ }^{6,43,44}$ another major way to reduce both PEMWE system and stack cost is by scaling-up WE production rate (Fig. 3c). Then, at economies of scale, the cost associated with PTLs and BPs drops significantly and stack cost is eventually dominated by MEA cost; this underlines the need for further studies on conductive substrates and on production scale-ups for PTLs and BPs. Despite such gains at the stack level, the 
precedent of scaling-up closed electrochemical storage systems is not directly transposable to open electrochemical conversion systems since system cost becomes increasingly dominated by BOP cost. ${ }^{37}$ Indeed, for 1MW PEMWE systems (Fig. 3b), a hundred-fold production increase (from 10 to 1000 units per year as shown in Fig. 3c) in combination with high-throughput, automated processes may lower the system cost from $561 \$ / \mathrm{kW}$ to $265 \$ / \mathrm{kW}$ and stack cost from $236 \$ / \mathrm{kW}$ to $69 \$ / \mathrm{kW}$, corresponding to some 42 and 26 $\%$ of system cost, respectively. ${ }^{40}$

This cost analysis is based on PEMWE technology, and it is yet unclear whether it holds true for AEMWEs, too, owing to the current lack of optimised/adequate membranes. Indeed, membranes developed for fuel cells or other uses are of limited suitability for WEs. ${ }^{41}$ This includes the commonly used Fumapem FAA series and Tokuyama Corporation A201 membranes; the latter has a limited conductivity $(\sim 40 \mathrm{mS} / \mathrm{cm}$ in deionized water) compared to protonic membranes $\left(\sim 100 \mathrm{mS} / \mathrm{cm}\right.$ for Nafion ${ }^{\circledR}$ in commercial protonic systems ${ }^{45}$ ), and suffers from limited mechanical and chemical stability in operating conditions. ${ }^{46-48}$ For WEs, material cost is thus inherently technology-dependent and certainly differs for AWE/AEMWE and PEMWE membranes as well as for catalysts, as discussed above. Nevertheless, it will ultimately not be the sole argument in assessing the scalability of a given technology. Rather, this will be largely determined by material abundancy and availability ${ }^{20}$ and by catalyst/MEA durability ${ }^{48}$.

The extent and pace of scaling technologies are inherently difficult to predict (Fig. 3d) with Li-ion batteries being at a much more mature stage than both fuel cell and water electrolyser systems and with fewer companies shaping the market. A strong push for WE systems in specific applications (e.g., PEMWE demonstrators for the chemical industry) could help the first stage of PEMWE scaling and open the way to higher production 
rates, which in turn results in a reduction of system cost especially at early stages of development, facilitating the deployment of large electrolyser plants ${ }^{8,11}$ and stimulating the demand for green hydrogen. Finally, translating learnings on production rate advances from PEM fuel cells (PEMFCs, now reaching thousands of units per year) to PEMWEs could help ensure a fast ramping of the production of water electrolysers. The cost projections for PEMWEs in Fig. 3d are very aggressive, but may eventually be reached due to technology spill-over from PEMFCs. ${ }^{11,49}$ Indeed, while fuel cells and water electrolysers are (and will remain) different in terms of chemistry, major gains can be expected by translating the knowledge acquired regarding component assembly and production scale up for plates.

\section{Emerging water electrolysers}

In summary, despite all our efforts at designing better catalysts and membranes, the future development and deployment of WEs at large scale is inherently dependent on the constraints associated with the current architectures and designs of both PEMWEs and AEMWEs. More precisely, the use of MEAs and PTLs largely explains that both the performances at the stack level and the price of these systems do not follow the recent findings at laboratory scale. To circumvent those constraints, engineering-inspired approaches have thus recently been aimed to develop new WE architectures and designs at the frontier between electrolyser and battery systems using concepts from both open and closed battery technology (i.e., redox flow cells and intercalation-based batteries). ${ }^{50}$ Such approaches were carried out with the expectation of relaxing operation and performance-limiting constraints placed upon WE components, but also to add functionality to WEs, following three main strategies (Fig. 4a). The first approach (A1, orange) uses 
membrane-less architectures and designs to circumvent delicate and costly membranes (see ref. ${ }^{51}$ for a comprehensive overview) so that ion transport occurs solely in liquid solution. This approach can be agnostic to the type of electrolyte, provided its ionic conductivity is suitable for the design, architecture and application, as shown in ref. ${ }^{52}$. The second and third approaches are directed at spatially and/or temporarily decoupling HER from OER, i.e., hydrogen from oxygen production. Indeed, while the OER provides electrons and protons to the HER, it produces oxygen which, while of value for specialty applications such as medical usage ${ }^{53}$, has limited industrial applicability compared to hydrogen. Thus, by decoupling these two reactions spatially, one could relax WE architecture- and design-specific constraints such as gas crossover and/or operating pressure. Additionally, if decoupled temporarily, it can separate electricity storage (electron generated through the OER) from fuel (i.e., hydrogen) generation. To do this, the second approach ( $\mathrm{A} 2$, pink) uses soluble electron and/or proton mediators so that charge transfer can be delocalised away from the electrodes, while the third one ( $A 3$, turquoise) uses auxiliary electrodes for integrating an energy storage capability, resulting in a battolyser. We observe that these three approaches have been successfully pursued separately or in combination, yielding new WE architectures and designs as shown in Fig. 4a for references $^{14,52,62,63,54-61}$.

To evaluate the impact of these approaches on the architectures and designs of WEs, we selected four examples of such emerging WEs (Fig. 4b). As an example, in ref. ${ }^{55}$, porous asymmetric conductive electrodes are used, serving as both PTLs and BPs directly coated with catalysts. Using such tailored electrodes was shown to separate hydrogen and oxygen gas in distinct headspaces with less than $1 \%$ gas crossover, by just controlling the 
geometry of these porous electrodes. Thus, a simple change in electrode geometry leads to membrane-less WEs (A1) with a significant impact on both WE architecture and design. A fundamentally different approach was adopted by Amstutz et al. ${ }^{61}$ for instance, where an integrated WE system with a redox flow-cell core using soluble redox mediators is demonstrated (A2). This system combines energy conversion and storage capability (though without auxiliary electrodes) with hydrogen production in separate catalytic beds, and disrupts traditional WE architectures. Chen et al. show ${ }^{60}$ a membrane-less, single-compartment WE system with hydrogen produced by coupling an HER electrode with a $\mathrm{NiOOH}$ electrode serving as a proton and electron reservoir which can be charged with a sacrificial $\mathrm{Zn}$ electrode. In contrast, Landman et $a .^{63}$ demonstrate a membraneless, two-compartment WE system with decoupled $\mathrm{O}_{2}$ and $\mathrm{H}_{2}$ production via auxiliary $\mathrm{Ni}(\mathrm{OH})_{2} / \mathrm{NiOOH}$ electrodes for energy storage. Both these studies combine the complementing approaches $A 1$ and $A 3$, while our survey shows that redundant approaches (A2 and A3) were not pursued in combination.

A common feature of the four WE devices mentioned above is that they eliminate the need for complex, degradation-prone MEAs, either by using membrane-less architectures (A1) or by temporally and/or spatially separating gas production (A2 or A3). Nevertheless, they remain open WE systems for which intrinsic properties of membranes/electrodes can hardly be scaled with extrinsic performances of the overall device, as extensively discussed previously. Indeed, MEA-free designs often suffer from large Ohmic drops, thus achieving lower current densities than AWEs. Unsurprisingly, data on the efficiency and gas production characteristics of emerging WEs (shown in Table $\mathbf{5 8}$ in the SI for the references listed in Fig. 4a) are difficult to compare, likely due to different definitions (see for example ref. ${ }^{64}$ for efficiency calculations). Based on limited experi- 
mental studies, the following trends can be extrapolated. For membrane-less WEs (A1) with no integration of soluble redox mediators or auxiliary electrodes, current densities as high as several hundreds of $\mathrm{mA} / \mathrm{cm}^{2}$ (without advective flow) to several $\mathrm{A} / \mathrm{cm}^{2}$ (with advective flow removing gaseous products) ${ }^{65}$ were achieved in lab-scale prototypes. However, when integrating soluble mediators or a proton-insertion electrode, the kinetics for charge transfer and/or proton exchange of these molecules/electrodes constrain current density, which might impede overall system performance. Furthermore, cell voltages $>2.3 \mathrm{~V}$ are found for membrane-less systems ${ }^{65,66}$ (compared to 1.8 to $2.2 \mathrm{~V}$ for PEMWEs at a current density of $\sim 2 \mathrm{~A} / \mathrm{cm}^{2}$ ). However, the use of soluble redox mediators and/or auxiliary electrodes will dictate the potential for the half-reaction providing electrons and protons to the HER and thus may result in potentials below this threshold. Finally, one significant improvement has recently been demonstrated by Dotan et al.; they achieved $50 \mathrm{~mA} / \mathrm{cm}^{2}$ at an average potential of $1.5 \mathrm{~V}$ (thus reaching a voltage efficiency of about $99 \%)$, by alternating HER at room temperature with self-discharge of an auxiliary $\mathrm{NiOOH}$ electrode for which oxygen evolution occurs spontaneously at $95{ }^{\circ} \mathrm{C} .{ }^{67}$ While such devices are energetically attractive, the long-term durability of using alternating flows of fluid and imposing a cyclic thermal strain on the catalysts is unclear.

Overall, these new WE devices, while promising, need to be benchmarked in preindustrialized conditions (i.e., at pilot-scale) with dimensions in the order of several dozens of $\mathrm{cm}^{2}$ of working area and a flux of reactant close to the one used, say, for PEMWEs so as to demonstrate performance stability over long timeframes. Under these conditions, limitations might arise for a number of reasons, such as the amount of dissolved redox molecules and the stability over cycling (both being observed in redox flow batteries), the loading and the stability of auxiliary electrodes ( $\mathrm{Ni}-\mathrm{Cd}$ and $\mathrm{Ni}-\mathrm{MH}$ batteries using 
$\mathrm{Ni}(\mathrm{OH})_{2}$ electrodes are known for their limited specific energy and important selfdischarge ${ }^{68}$ ), or the required $\mathrm{H}_{2}$ output pressure. With MEA-based devices, the production of pressurised hydrogen is at approximately the same pressure ( 30 bar) as with steam methane reforming. To reach this pressure level, membrane-less devices would require an additional three-step compression system corresponding to $\sim 2.8 \mathrm{kWh} / \mathrm{kg}$ of $\mathrm{H}_{2}$ (translating into an additional $\sim 0.3 \$ / \mathrm{kg}$ of $\mathrm{H}_{2}$, see Discussion $\mathbf{S 9}$ in the $\mathrm{SI}$ ), drastically limiting cost efficiency and certainly constrain their usability for onsite applications. Even if their performance metrics fall short in comparison with industry-sized PEMWEs, their efficiency is encouraging. Due to the modified operational conditions, in some systems, voltage efficiency $>60 \%$ and Faradaic efficiency $>95 \%$ are observed for hydrogen production. Several recent start-ups aim to develop and assess the viability of new WEs design relying on the overcharge of $\mathrm{Ni}$-Fe batteries ${ }^{69}$ (aiming at high energy efficiency and volumetric energy), the use of a zinc auxiliary electrode ${ }^{70}$ (aiming at high-pressure hydrogen production) or the use of flow-through electrodes combined with a membrane-less architecture ${ }^{71}$. 


\section{Conclusion}

Novel architectures and designs address some critical aspects of WEs technologies, while inherently remaining open systems (energy storage functionality notwithstanding), in which performance and cost are severely limited by constraints associated with BOP equipment like liquid and gas management and power electronics. Uncertainty also remains as to the devices' ability to cope with the variability of renewable energies. They might fall short of securing a steady production of green hydrogen for industrial applications and be better suited for the complementary and local production of hydrogen. The major impact of modifying WE architectures and designs may be in enabling the development and rapid deployment of cost-effective, durable and scalable WEs for partialload production of sustainable hydrogen by circumventing the use of MEAs and/or PTLs and BPs. Though most of these devices are still in their infancy and far removed from scaling-up and mass deployment, it is crucial to benchmark them in their present stage and to compare them against state-of-the-art AWEs and PEMWEs. Indeed, assessing the new architectures' and designs' actual potentiality for large-scale applications at a very early stage of their development is essential for focussing research efforts onto the most critical aspects of their technologies. In turn, diversifying the available architectures and designs of WE devices is a sensible strategy for extending the spectrum of application scenarios of efficient hydrogen production. The new devices may be used to complement classical PEMWEs and AEMWEs, which are still the technologies of choice for scaling up of green hydrogen production for industrial applications.

We believe that material scientists must bear in mind such engineering aspects when designing better electrodes/catalysts. Integrating engineering strategies in our research approaches, though, is no substitute for focusing large research efforts towards answer- 
ing fundamental questions at the heart of catalyst and/or membrane development. A clear distinction between the goal of advancing our fundamental understanding of the complex processes occurring at electrified interfaces (materials science with long-term gains) and that of developing better industrial components of WEs (engineering with short- and long-term gains) is important. The translation from laboratory findings into large-scale production (i.e., from materials science to engineering and manufacturing) is crucial in making WE technology the next electrochemical success story and a core technology in a sustainable, low-emission energy mix. To this end, the future deployment of better WE architectures and designs necessitates a stronger collaboration between material scientists, electrochemists and engineers. 


\section{Alphabetical list of acronyms}

AEMWE Anion exchange membrane water electrolyser

AWE Alkaline water electrolyser

BOP Balance of plant

BP Bipolar plate

EP End plate

HER Hydrogen evolution reaction

LIB Lithium ion battery

MEA Membrane electrode assembly

OER Oxygen evolution reaction

PEMWE Proton exchange membrane water electrolyser

PTL Porous transport layer

TEC Three electrode configuration 


\section{Box 1 | Interrelatedness of water electrolyser components, performance and cost.}

For today's commercially available low-temperature WEs - alkaline water electrolysis (AWE), the older and more mature technology, as well as for the more recent proton exchange membrane water electrolysis (PEMWE) technology - the very core of any device is its membrane or diaphragm, which ensures ion transport towards the electrodes on which catalysts are loaded. In classical AWEs (Fig. S1a in the supplementary information, $\mathrm{SI}$ ), the $\mathrm{Ni}$-based electrodes are simply immersed in an alkaline solution and are separated by a diaphragm, preventing the cross-diffusion of gases. In contrast, for PEMWEs (Fig. S1b in the SI), the membrane and the electrodes are combined in a membrane electrode assembly (MEA, Fig. 2a). The main research efforts are directed at improving the normalised activity of PEMWE catalysts, ${ }^{15,20}$ though the MEAs' properties can hardly be directly extrapolated from the intrinsic properties of the membrane and the catalystloaded electrodes. The catalyst particles are held together by an ion-conducting polymer, i.e., an ionomer; playing an equivalent to the binder polymers for Li-ion cell electrodes. The most commonly used ionomer is $\operatorname{Nafion}^{\circledR}$, a sulfonate ionomer which is also used as the PEMWE membrane. The MEA is sandwiched between porous transport layers (PTLs, made from porous titanium (Ti) and carbon paper) that ensure the mass transport of water towards the electrodes and of gas away. The PTLs themselves are connected to bipolar plates (BPs, made from Ti) serving as current collectors with inlets for liquid and outlets for gas. Together, the PTLs and BPs have a total thickness of several millimetres and are pressed together with end plates (EPs). For anion exchange membrane water electrolysers (AEMWEs, Fig. S1c in the SI), the diaphragm commonly used for AWE is replaced by an anion conducting membrane (still under development by for example Fu- 
matech $^{72}$ or Dioxide Materials ${ }^{73}$ ) and used with a PEMWE stack architecture. This technology is still in its infancy and - with matching membranes and ionomers - has the potential to achieve performance characteristics suitable for rapid industrial deployment, with few commercial AEMWE devices already existing (e.g., by Enapter ${ }^{74}$ and Proton Onsite $^{75,76}$ ). For AEMWEs, the main research efforts are directed either toward reaching a better fundamental understanding about, for instance, the effect of solvent-catalyst interface on reaction kinetics, ${ }^{22,77-79}$ or to the development of stable membranes ${ }^{41}$.

A water electrolyser's smallest operating unit is a cell, which is the MEA sandwiched by the PTLs and BPs. A half-cell (symmetric in terms of components but not materials) is depicted on the left side. Stack performance is primarily dependent on durability (chemical and mechanical integrity of materials and components), efficiency (suitability of materials and of component interfaces) and upscaling (dimension of active area). These parameters are dominated by the properties of the MEA, PTLs and BPs as indicated by the blue and purple circles. Stack performance along with operating conditions (such as applied current density, voltage, solution feed rate and purity, and dynamics) define system performance, i.e., of a multitude of cells and/or stacks with surrounding control and processing equipment.

Stack cost depends on material cost (membrane, ionomer, platinum group metals for PEMWE catalysts, titanium for PTLs and BPs), scalability (availability of raw and processed materials) and manufacturing and integration (advances in manufacturing techniques such as 3D printing and in merging of components). It is dominated by the intrinsic properties of the membrane, catalysts, PTLs and BPs as indicated by the yellow, magenta and purple circles. For today's systems, the PTLs and BPs made from Ti plates make 
up more than a third of the stack $\operatorname{cost}^{41,80}$ (Fig. 3a); they are up to three times more expensive than their stainless steel, copper or aluminium counterparts. ${ }^{39}$

A water electrolyser system with a multitude of cells and/or stacks requires balance of plant (BOP) equipment ensuring hydrogen processing, deionised water circulation and power supply. System cost (in $\$ / \mathrm{kW}$ ) depends both on stack cost and BOP cost (their weighting depending on system size, production volumes and manufacturing advances) as well as system performance. The cost of hydrogen production (in $\$ / \mathrm{kg}$ of $\mathrm{H}_{2}$ ) depends on operating expenditure (OPEX: primarily dominated by electricity cost) and capital expenditure (CAPEX: system cost, installation cost and other investment cost), and will ultimately determine the extent of future market penetration of water electrolyser systems. The depicted relationship between components, performance and cost does not explicitly address technology, operating mode, stack size, system size, production rates and manufacturing advances. This is because these parameters shift the proportion of material/component impact on performance and cost as discussed in the main text and shown in Fig. 3. 


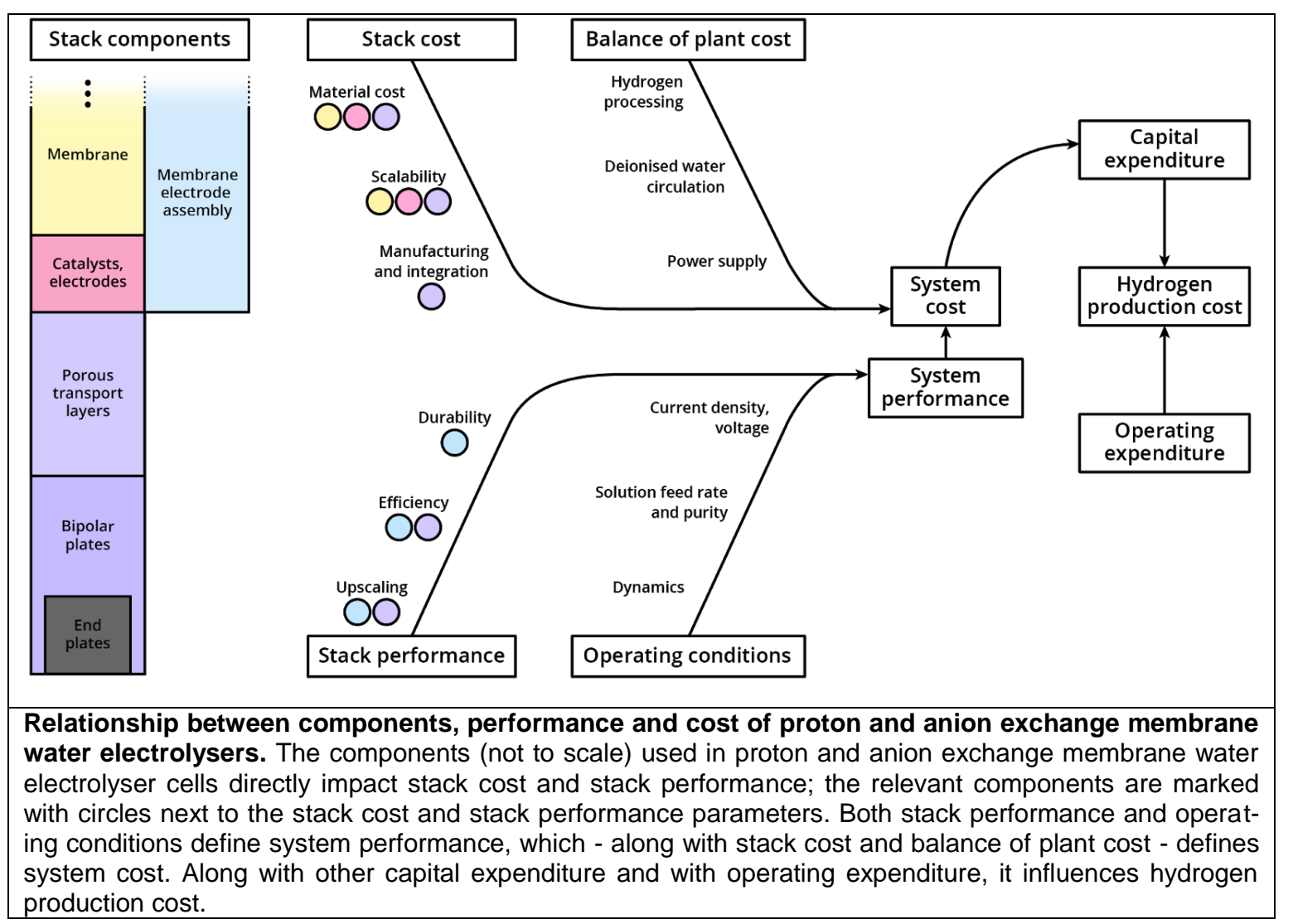




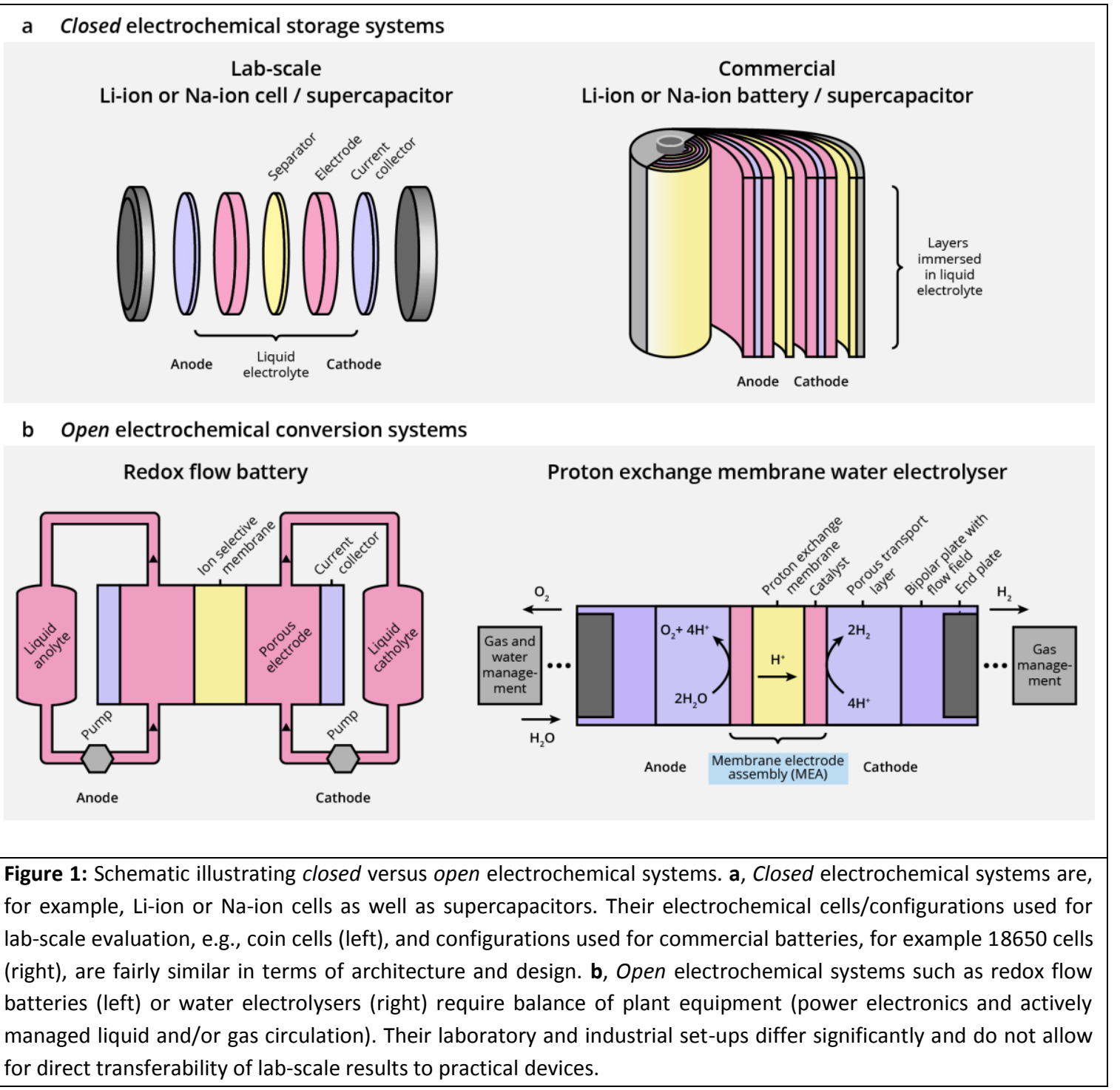




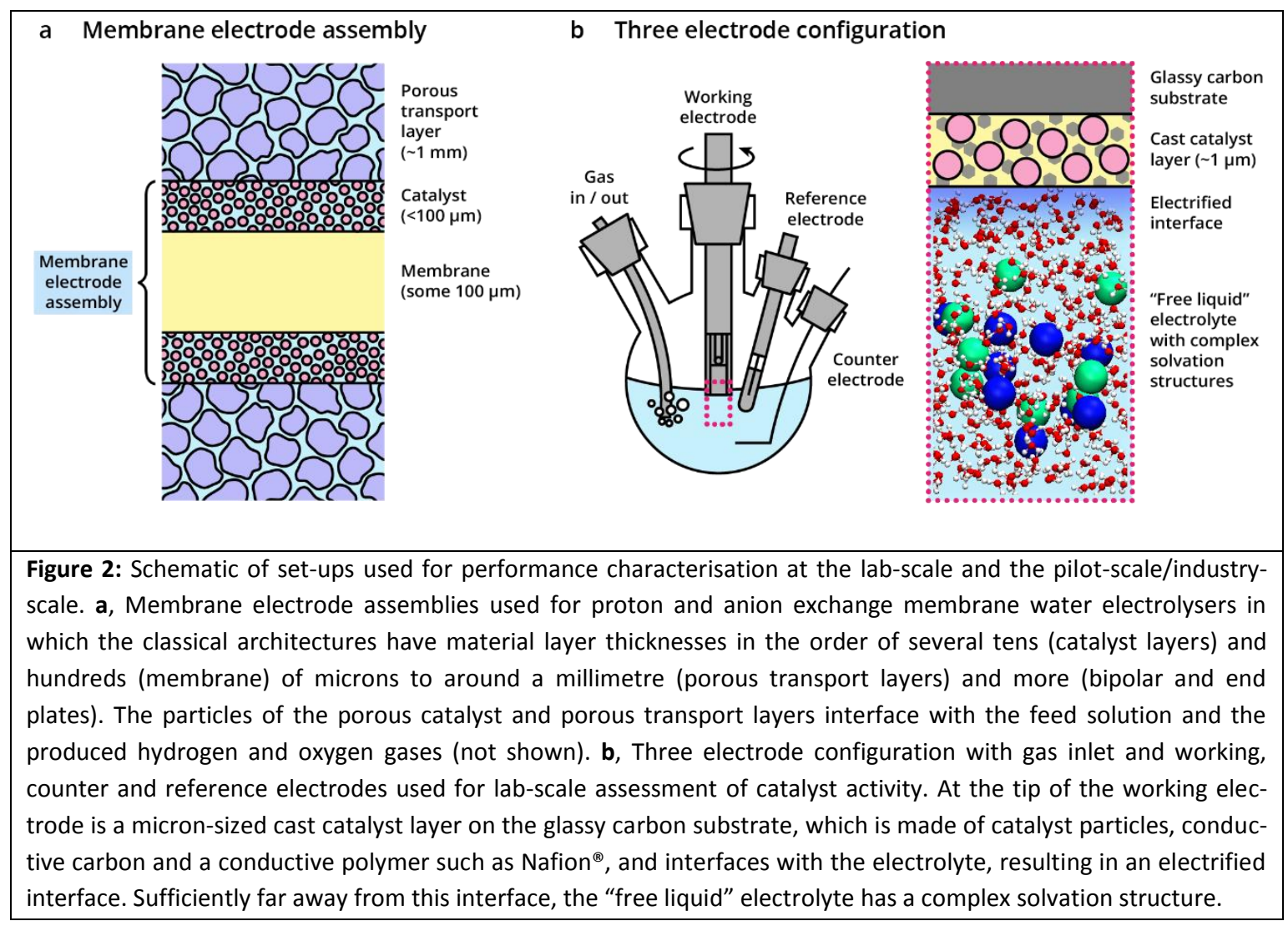




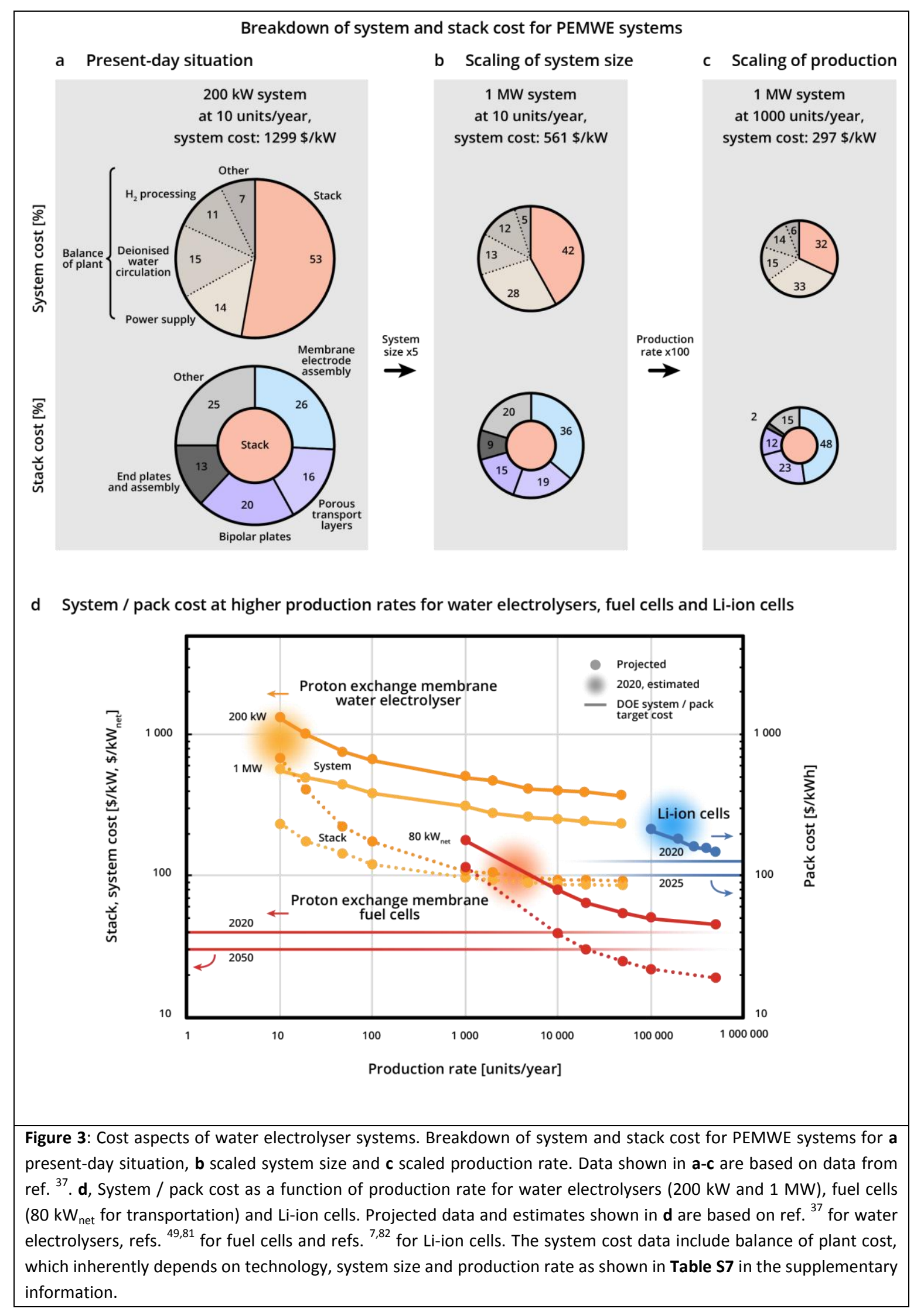




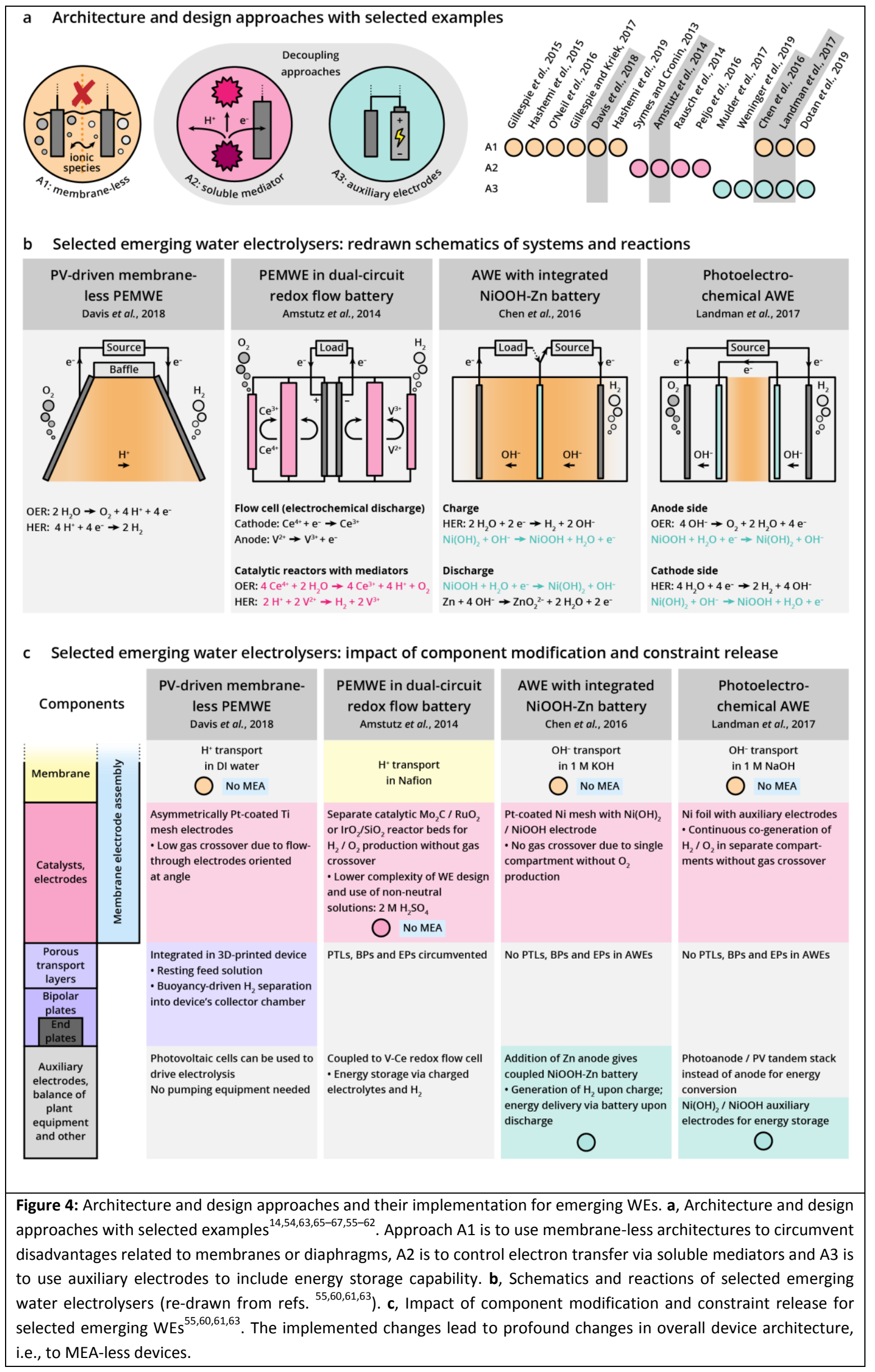




\section{References}

1. Le Quéré, C. et al. Global Carbon Budget 2018. Earth Syst. Sci. Data 10, 2141-2194 (2018).

2. International Renewable Energy Agency. Global energy transformation: A roadmap to 2050. (2019).

3. International Energy Agency. Global Energy and CO2 Status Report. (2017). doi:10.4324/9781315252056

4. European Commission. Energy prices and cost in Europe. (European Commission, 2019).

5. Winter, M., Barnett, B. \& Xu, K. Before Li Ion Batteries. Chem. Rev. 118, 1143311456 (2018).

6. Curry, C. Lithium-ion battery costs and market. (Bloomberg New Energy Finance, 2017).

7. Pillot, C. Impact of the XEV Market growth on Lithium-Ion Batteries and Raw Materials Supply 2019-2030. (Avicenne Energy, 2020).

8. International Energy Agency. The Future of Hydrogen. (2019). doi:10.1016/S14642859(12)70027-5

9. International Renewable Energy Agency. Hydrogen: A renewable energy perspective. (2019).

10. van Hulst, N. Commentary: The clean hydrogen future has already begun. (2019). Available at: https://www.iea.org/newsroom/news/2019/april/the-cleanhydrogen-future-has-already-begun.html. (Accessed: 25th November 2019)

11. Gül, T., Fernandez Pales, A. \& Paoli, L. Batteries and hydrogen technology: keys for a clean energy future. (2020). 
12. Nørskov, J. K. et al. Research needs towards sustainable production of fuels and chemicals. (ENERGY-X, 2019).

13. Battolyser. Available at: https://battolyser.com. (Accessed: 13th December 2019)

14. Weninger, B. M. H. \& Mulder, F. M. Renewable Hydrogen and Electricity Dispatch with Multiple Ni-Fe Electrode Storage. ACS Energy Lett. 4, 567-571 (2019).

15. Bernt, M. et al. Current Challenges in Catalyst Development for PEM Water Electrolyzers. Chemie Ing. Tech. 90, (2020).

16. Faustini, M. et al. Hierarchically Structured Ultraporous Iridium-Based Materials: A Novel Catalyst Architecture for Proton Exchange Membrane Water Electrolyzers. Adv. Energy Mater. 9, 1802136 (2019).

17. Wu, T. et al. Iron-facilitated dynamic active-site generation on spinel $\mathrm{CoAl}_{2} \mathrm{O}_{4}$ with self-termination of surface reconstruction for water oxidation. Nat. Catal. 2, 763772 (2019).

18. King, L. A. et al. A non-precious metal hydrogen catalyst in a commercial polymer electrolyte membrane electrolyser. Nat. Nanotechnol. 14, 1071-1074 (2019).

19. Seh, Z. W. et al. Combining theory and experiment in electrocatalysis: Insights into materials design. Science. 355, (2017).

20. Kibsgaard, J. \& Chorkendorff, I. Considerations for the scaling-up of water splitting catalysts. Nat. Energy 4, 430-433 (2019).

21. Inaba, M. et al. Benchmarking high surface area electrocatalysts in a gas diffusion electrode: measurement of oxygen reduction activities under realistic conditions. Energy Environ. Sci. 11, 988-994 (2018).

22. Wei, C. et al. Recommended Practices and Benchmark Activity for Hydrogen and Oxygen Electrocatalysis in Water Splitting and Fuel Cells. Adv. Mater. 31, 1806296 
(2019).

23. Suntivich, J., Gasteiger, H. A., Yabuuchi, N. \& Shao-Horn, Y. Electrocatalytic Measurement Methodology of Oxide Catalysts Using a Thin-Film Rotating Disk Electrode. J. Electrochem. Soc. 157, B1263-B1268 (2010).

24. Debe, M. K. Electrocatalyst approaches and challenges for automotive fuel cells. Nature 486, 43-51 (2012).

25. Kroschel, M., Bonakdarpour, A., Kwan, J. T. H., Strasser, P. \& Wilkinson, D. P. Analysis of oxygen evolving catalyst coated membranes with different current collectors using a new modified rotating disk electrode technique. Electrochim. Acta 317, 722-736 (2019).

26. Bender, G. et al. Initial approaches in benchmarking and round robin testing for proton exchange membrane water electrolyzers. Int. J. Hydrogen Energy 44, 91749187 (2019).

27. Ayers, K. Benchmarking Advanced Water Splitting Technologies: Best Practices in Materials Characterization. (Energy Materials Network, U.S. Department of Energy, 2019).

28. Colli, A. N., Girault, H. H. \& Battistel, A. Non-precious electrodes for practical alkaline water electrolysis. Materials (Basel). 12, 1-17 (2019).

29. Weiß, A. et al. Impact of Intermittent Operation on Lifetime and Performance of a PEM Water Electrolyzer. J. Electrochem. Soc. 166, F487-F497 (2019).

30. Debe, M. K. et al. Initial Performance and Durability of Ultra-Low Loaded NSTF Electrodes for PEM Electrolyzers. J. Electrochem. Soc. 159, K165-K176 (2012).

31. Bock, R. et al. Measuring the thermal conductivity of membrane and porous transport layer in proton and anion exchange membrane water electrolyzers for 
temperature distribution modeling. Int. J. Hydrogen Energy 1-19 (2019).

doi:10.1016/j.ijhydene.2019.01.013

32. Wang, L. et al. Electrochemically converting carbon monoxide to liquid fuels by directing selectivity with electrode surface area. Nat. Catal. 2, 702-708 (2019).

33. Andersen, S. Z. et al. A rigorous electrochemical ammonia synthesis protocol with quantitative isotope measurements. Nature 570, 504-508 (2019).

34. Wakerley, D. et al. Bio-inspired hydrophobicity promotes $\mathrm{CO}_{2}$ reduction on a $\mathrm{Cu}$ surface. Nat. Mater. 18, 1222-1227 (2019).

35. Weng, L.-C., Bell, A. T. \& Weber, A. Z. Modeling gas-diffusion electrodes for $\mathrm{CO}_{2}$ reduction. Phys. Chem. Chem. Phys. 20, 16973-16984 (2018).

36. Burdyny, T. \& Smith, W. A. $\mathrm{CO}_{2}$ reduction on gas-diffusion electrodes and why catalytic performance must be assessed at commercially-relevant conditions. Energy Environ. Sci. 12, 1442-1453 (2019).

37. Mayyas, A. et al. Manufacturing Cost Analysis for Proton Exchange Membrane Water Electrolyzers. (National Renewable Energy Laboratory, 2019).

38. Fritz, K. E., Beaucage, P. A., Matsuoka, F., Wiesner, U. \& Suntivich, J. Mesoporous titanium and niobium nitrides as conductive and stable electrocatalyst supports in acid environments. Chem. Commun. 53, 7250-7253 (2017).

39. Gago, A. S. et al. Protective coatings on stainless steel bipolar plates for proton exchange membrane (PEM) electrolysers. J. Power Sources 307, 815-825 (2016).

40. Ayers, K., Capuano, C. B. \& Anderson, E. B. Recent Advances in Cell Cost and Efficiency for PEM-Based Water Electrolysis. ECS Trans. 41, 15-22 (2012).

41. Ayers, K. et al. Perspectives on Low-Temperature Electrolysis and Potential for Renewable Hydrogen at Scale. Annu. Rev. Chem. Biomol. Eng. 10, 219-239 (2019). 
42. Bernt, M., Siebel, A. \& Gasteiger, H. A. Analysis of Voltage Losses in PEM Water Electrolyzers with Low Platinum Group Metal Loadings. J. Electrochem. Soc. 165, F305-F314 (2018).

43. Schmuch, R., Wagner, R., Hörpel, G., Placke, T. \& Winter, M. Performance and cost of materials for lithium-based rechargeable automotive batteries. Nat. Energy 3, 267-278 (2018).

44. Pillot, C. The Rechargeable Battery Market and Main Trends 2018-2030. (Avicenne Energy, 2019).

45. Lehner, M., Tichler, R., Steinmüller, H. \& Koppe, M. Water Electrolysis. in Powerto-Gas: Technology and Business Models 19-39 (Springer International Publishing, 2014). doi:10.1007/978-3-319-03995-4_3

46. Yanagi, H. \& Fukuta, K. Anion exchange membrane and ionomer for alkaline membrane fuel cells (AMFCs). ECS Trans. 16, 257-262 (2008).

47. Varcoe, J. R. et al. Anion-exchange membranes in electrochemical energy systems. Energy Environ. Sci. 7, 3135-3191 (2014).

48. Abbasi, R. et al. A Roadmap to Low-Cost Hydrogen with Hydroxide Exchange Membrane Electrolyzers. Adv. Mater. 31, 1805876 (1-14) (2019).

49. Whiston, M. M. et al. Expert assessments of the cost and expected future performance of proton exchange membrane fuel cells for vehicles. Proc. Natl. Acad. Sci. U. S. A. 116, 4899-4904 (2019).

50. You, B. \& Sun, Y. Innovative Strategies for Electrocatalytic Water Splitting. Acc. Chem. Res. 51, 1571-1580 (2018).

51. Esposito, D. V. Membraneless Electrolyzers for Low-Cost Hydrogen Production in a Renewable Energy Future. Joule 1, 651-658 (2017). 
52. H. Hashemi, S. M., Modestino, M. A. \& Psaltis, D. A membrane-less electrolyzer for hydrogen production across the pH scale. Energy Environ. Sci. 8, 2003-2009 (2015).

53. Kato, T., Kubota, M., Kobayashi, N. \& Suzuoki, Y. Effective utilization of by-product oxygen from electrolysis hydrogen production. Energy 30, 2580-2595 (2005).

54. O’Neil, G. D., Christian, C. D., Brown, D. E. \& Esposito, D. V. Hydrogen Production with a Simple and Scalable Membraneless Electrolyzer. J. Electrochem. Soc. 163, F3012-F3019 (2016).

55. Davis, J. T., Qi, J., Fan, X., Bui, J. C. \& Esposito, D. V. Floating membraneless PVelectrolyzer based on buoyancy-driven product separation. Int. J. Hydrogen Energy 43, 1224-1238 (2018).

56. Hashemi, S. M. H. et al. A versatile and membrane-less electrochemical reactor for the electrolysis of water and brine. Energy Environ. Sci. 12, 1592-1604 (2019).

57. Symes, M. D. \& Cronin, L. Decoupling hydrogen and oxygen evolution during electrolytic water splitting using an electron-coupled-proton buffer. Nat. Chem. 5, 403-409 (2013).

58. Rausch, B., Symes, M. D., Chisholm, G. \& Cronin, L. Decoupled catalytic hydrogen evolution from a molecular metal oxide redox mediator in water splitting. Science (80-. ). 345, 1326-1330 (2014).

59. Mulder, F. M., Weninger, B. M. H., Middelkoop, J., Ooms, F. G. B. \& Schreuders, H. Efficient electricity storage with a battolyser, an integrated Ni-Fe battery and electrolyser. Energy Environ. Sci. 10, 756-764 (2017).

60. Chen, L., Dong, X., Wang, Y. \& Xia, Y. Separating hydrogen and oxygen evolution in alkaline water electrolysis using nickel hydroxide. Nat. Commun. 7, 1-8 (2016). 
61. Amstutz, V. et al. Renewable hydrogen generation from a dual-circuit redox flow battery. Energy Environ. Sci. 7, 2350-2358 (2014).

62. Peljo, P. et al. All-vanadium dual circuit redox flow battery for renewable hydrogen generation and desulfurisation. Green Chem. 19, 1785-1797 (2016).

63. Landman, A. et al. Photoelectrochemical water splitting in separate oxygen and hydrogen cells. Nat. Mater. 16, 646-652 (2017).

64. Tsotridis, G. \& Pilenga, A. EU harmonised terminology for low temperature water electrolysis for energy storage applications. (Publications Office of the European Union, 2018). doi:10.2760/138987

65. Gillespie, M. I., Van Der Merwe, F. \& Kriek, R. J. Performance evaluation of a membraneless divergent electrode-flow-through (DEFT) alkaline electrolyser based on optimisation of electrolytic flow and electrode gap. J. Power Sources 293, 228-235 (2015).

66. Gillespie, M. I. \& Kriek, R. J. Hydrogen production from a rectangular horizontal filter press Divergent Electrode-Flow-Through (DEFT ${ }^{\mathrm{TM}}$ ) alkaline electrolysis stack. J. Power Sources 372, 252-259 (2017).

67. Dotan, H. et al. Decoupled hydrogen and oxygen evolution by a two-step electrochemical-chemical cycle for efficient overall water splitting. Nat. Energy 4, 786-795 (2019).

68. Palacín, M. R. \& De Guibert, A. Batteries: Why do batteries fail? Science. 351, $1253292(1-7)(2016)$.

69. Battolyser B.V. Available at: https://www.battolyserbv.com. (Accessed: 7th January 2020)

70. ERGOSUP. Available at: https://www.ergosup.com/electrolyseur-sous-pression/. 
(Accessed: 7th January 2020)

71. Hydrox Holdings Ltd. Available at: https://hydroxholdings.co.za. (Accessed: 22nd April 2020)

72. Fumatech. Available at: https://www.fumatech.com. (Accessed: 22nd April 2020)

73. Dioxide Materials. Available at: https://dioxidematerials.com. (Accessed: 22nd April 2020)

74. Pavel, C. C. et al. Highly Efficient Platinum Group Metal Free Based MembraneElectrode Assembly for Anion Exchange Membrane Water Electrolysis. Angew. Chemie - Int. Ed. 126, 1402-1405 (2014).

75. Parrondo, J. et al. Degradation of anion exchange membranes used for hydrogen production by ultrapure water electrolysis. RSC Adv. 4, 9875-9879 (2014).

76. Gardner, G. et al. Structural Basis for Differing Electrocatalytic Water Oxidation by the Cubic, Layered and Spinel Forms of Lithium Cobalt Oxides. Energy Environ. Sci. 9, 184-192 (2016).

77. Yoon, Y., Yan, B. \& Surendranath, Y. Suppressing Ion Transfer Enables Versatile Measurements of Electrochemical Surface Area for Intrinsic Activity Comparisons. J. Am. Chem. Soc. 140, 2397-2400 (2018).

78. Dubouis, N. \& Grimaud, A. The hydrogen evolution reaction: From material to interfacial descriptors. Chem. Sci. 10, 9165-9181 (2019).

79. Garcia, A. C., Touzalin, T., Nieuwland, C., Perini, N. \& Koper, M. T. M. Enhancement of Oxygen Evolution Activity of Nickel Oxyhydroxide by Electrolyte Alkali Cations. Angew. Chemie - Int. Ed. 58, 12999-13003 (2019).

80. Babic, U., Suermann, M., Büchi, F. N., Gubler, L. \& Schmidt, T. J. Critical ReviewIdentifying Critical Gaps for Polymer Electrolyte Water Electrolysis Development. J. 
Electrochem. Soc. 164, F387-F399 (2017).

81. Wilson, A., Kleen, G. \& Papageorgopoulos, D. Fuel Cell System Cost - 2017. (US Department Of Energy, 2017).

82. Slowik, P., Pavlenko, N. \& Lutsey, N. Assessment of next-generation electric vehicle technologies. (The International Council on Clean Transportation, 2016).

\section{Acknowledgements}

The authors acknowledge financial support from the ANR MIDWAY project (project ID: ANR-17-CE05-0008), from the French national network "Réseau sur le Stockage Electrochimique de l'Energie" (RS2E) FR CNRS 3459 and from the Laboratory of Excellence program STORE-EX (ANR 10-LABX-0076). They thank Nicolas Dubouis for his contribution to Figure $2 b$, Claire Ferchaud for useful discussions regarding hydrogen pressurisation and Jean-Marie Tarascon, Nicolas Dubouis, Romain Dugas and Betar M. Gallant for critical examination of the manuscript in its early stage.

\section{Author contributions}

M.F.L. and A.G. contributed equally to conceiving, developing and writing the manuscript.

\section{Competing interests}

The authors declare no competing interests. 